\title{
DAMPING CONTROL OF MEMS DEVICES USING STRUCTURAL DESIGN APPROACH
}

\author{
C.-L. Chen and J. Jason Yao \\ Rockwell Science Center \\ 1049 Camino Dos Rios \\ Thousand Oaks, CA 91360
}

\begin{abstract}
The transient responses or dynamics of MEMS parallelplate, squeeze type devices have been simulated numerically in the present work. Emphasis is given to the study of the microfluidynamic damping effect by solving the Reynolds equation with a slip boundary condition at the structure surfaces. This approach couples the micro-fluidynamics into the dynamic simulation of MEMS devices in a CPU efficient way, and allows a damping effect simulation at every time step. The computed results indicate that the damping behavior can be altered using a structural design approach which employs structural modification by creating various "holes" in the paddle-like plate structure and by varying the hole distributions on the paddle. The transient time for one of the test structures, for example, is reduced from $9 \mathrm{~ms}$ in the without-hole case to $70 \mu \mathrm{s}$ in the with-hole case. These simulation results have been qualitatively supported by experimental data.
\end{abstract}

\section{INTRODUCTION}

For physical and cost reasons, MEMS devices are seldom operated in high vacuum. The performance of MEMS devices is thus strongly affected by the surrounding fluids, e.g., air. Consequently, understanding the fluid behavior at micro scale, modeling the fluid effects, coupling it into the dynamic system, and tracing the dynamic response are crucial steps in the design of MEMS devices for practical applications. Several works have addressed fluid effects on various MEMS devices with various numerical approaches [1-4]. The Direct Simulation Monte Carlo (DSMC) type approach is still very CPU intensive. In this work, we present a method of computing microfluidynamic behavior for MEMS parallel-plate, squeeze type devices to obtain the damping load by solving the Reynolds equation with slip-flow consideration. The damping force acting on the cantilevered paddle structure is a function of the paddle velocity, the gap, the Knudsen number $K n=\lambda / \hbar$ (where $\lambda$ is the mean free path and $h$ is the fluid film thickness), the surrounding fluid properties, and the paddle configuration. Our approach couples the micro-fluidynamics into the dynamic simulation of MEMS devices in a CPU efficient way, and is able to provide a damping effect simulation at every time step using a single workstation. Here we focus on examining the resulting effects of various structural changes on the dynamics of electrostatically driven plate structures. The change of structural design is made by creating "holes" on the moving paddle and by changing the hole configuration on the paddle.

\section{ANALYSIS}

The model is shown in Fig. 1. The gap $h$ between the paddle and the substrate is assumed to be much smaller than the paddle dimensions in the $x$ and $y$ directions. The paddle is supported by a spring (not shown in Fig. 1) and can move vertically in the $z$ direction. The gas flow is assumed laminar and primarily viscous. The relationship between pressure and density at any point in the gas film is described by a polytropic process with exponent $n: P / \rho^{n}=$ constant.

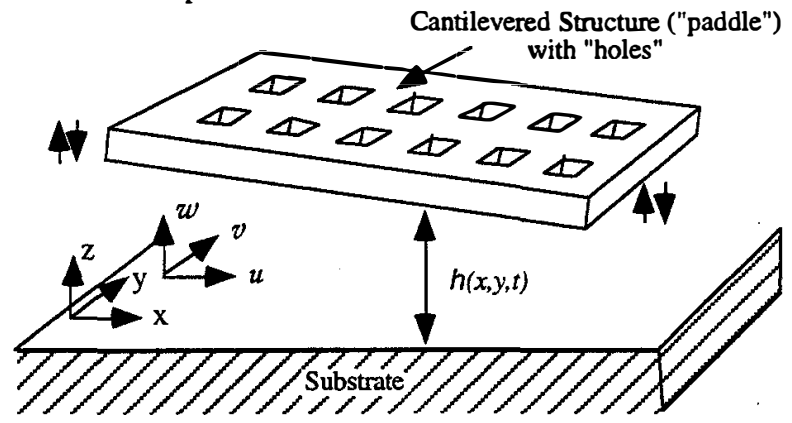

Figure 1. A schematic illustration showing the model to be simulated.

Suppose that function $F(x, y, z, t)=0$ represents the equation for a moving boundary surface, that is

$$
F=\boldsymbol{z}-h(x, y, t)=0
$$

When the solid boundary is in motion, the fluid velocity component normal to the boundary surface equals the velocity component of the surface normal to itself, but the flow is still allowed to slip in the tangential direction along the boundary surface. That is,

$$
\mathbf{V} \cdot \mathbf{n}=\mathbf{U}_{\mathbf{b}} \cdot \mathbf{n}
$$

where $\mathbf{n}$ is the unit vector normal to the surface, $\mathbf{V}(u, v, w)$ is the velocity of the fluid particle at the paddle boundary and $\mathbf{U}_{\mathbf{b}}$ denotes the velocity of the points on the boundary with which the fluid particle is in contact. Using the above equations, we can obtain the vertical component, $w$, of the velocity vector at the moving boundary $h(x, y, t)$ as

$$
w=u \frac{\partial h}{\partial x}+v \frac{\partial h}{\partial y}+\frac{\partial h}{\partial t}
$$

which is the general kinematic boundary condition equation. Using this equation and integrating compressible continuity equation with respect to $z$ and applying Leibnitz's theorem, we obtain

$$
\frac{\partial \rho h}{\partial t}+\frac{\partial}{\partial x} \int_{0}^{h} \rho u \mathrm{~d} z+\frac{\partial}{\partial y} \int_{0}^{h} \rho v \mathrm{~d} z=0
$$

For $x$ momentum (also similarly for $y$ momentum), under the assumptions of incompressibility, constant pressure across the fluid film in the squeeze direction, and small Reynolds number and fluid inertia, the above equation may be approximated to

$$
\frac{\mathrm{d} p}{\mathrm{~d} x}=\mu \frac{\mathrm{d}^{2} u}{\mathrm{~d} z^{2}}
$$

where $\mu$ is the viscosity. Using slip flow boundary conditions[5], $u=U_{\mathrm{s}}$ at $z=0$, and $u=-U_{\mathrm{s}}$ at $z=h$, and

$$
U_{\mathrm{s}}=\left.\lambda \alpha \frac{\mathrm{d} u}{\mathrm{~d} z}\right|_{\text {wall }}, \alpha=\frac{2-d}{d}
$$


where $\lambda$ is the mean free path, and $d$ is the diffuse reflection coefficient, the velocity profile becomes

$$
u=\frac{h^{2}}{2 \mu} \frac{\mathrm{d} p}{\mathrm{~d} x}\left(\overline{\mathrm{z}}^{2}-\overline{\mathrm{z}}-\alpha \mathscr{X}_{\mathrm{J}}\right), \overline{\mathrm{z}}=\frac{z}{h}
$$

Similar procedure can be repeated for $v$ in the $y$ direction. Therefore, Eq. 4 becomes

$$
\begin{aligned}
12 \mu \frac{\partial p}{\partial t} \frac{\frac{1}{\mathrm{n}}}{\partial t}-\frac{\mathrm{n}}{\mathrm{n}+1} & \left(\frac{\partial}{\partial x}\left(h^{3}\left(1+6 \alpha \mathcal{K}_{2}\right) \frac{\partial}{\partial x} p^{\frac{\mathrm{n}+1}{\mathrm{n}}}\right)\right. \\
+ & \left.\frac{\partial}{\partial y}\left(h^{3}\left(1+6 \alpha \mathcal{K}_{2}\right) \frac{\partial}{\partial y} p^{\frac{\mathrm{n}+1}{\mathrm{n}}}\right)\right)=0
\end{aligned}
$$

If $h=h(t)$ only in the parallel plate case (Special Case \#1), the equation can be further simplified to

$$
\begin{aligned}
h \frac{\partial p^{\frac{1}{n}}}{\partial t}-\frac{h^{3}\left(1+6 \alpha \mathcal{K}_{\Omega}\right)}{12 \mu} & \frac{n}{n+1}\left(\frac{\partial^{2}}{\partial x^{2}} p^{\frac{n+1}{n}}\right. \\
& \left.+\frac{\partial^{2}}{\partial y^{2}} p^{\frac{n+1}{n}}\right)=-p^{\frac{1}{n}} \frac{\partial h}{\partial t}
\end{aligned}
$$

For small pressure fluctuation (Special Case \#2), pressure may be approximated as $p=p_{\mathrm{a}}+p^{\prime}$, in which $p_{\mathrm{a}}$ is the ambient pressure and $p^{\prime}$ is the pressure perturbation. In the isothermal process $(\mathrm{n}=1)$, the equation can be written as

$$
p_{\mathrm{a}} \frac{\partial h}{\partial t}+h \frac{\partial p^{\prime}}{\partial t}-\frac{h^{3}\left(1+6 \alpha \mathcal{K}_{2}\right)}{12 \mu} p_{\mathrm{a}}\left(\frac{\partial^{2} p^{\prime}}{\partial x^{2}}+\frac{\partial^{2} p^{\prime}}{\partial y^{2}}\right)=0
$$

Under the condition that $\boldsymbol{h} \partial \partial^{\prime} / \mathrm{t}$ is negligible (Special Case \#3), the equation is a linear Poisson's equation in which the forcing term strength is proportional to $(\partial h / \partial t)\left(1 / h^{3}\right)$. Therefore, for each configuration, Eq. 10 needs to be solved only once for a unit forcing term. The rest of the solutions can be obtained by superposition.

\section{NUMERICAL APPROACH}

Here we define an unknown variable $q$ for $p^{(1 / n)}$ and solve for $q$ numerically. The general case Eq. 8 can be rewritten as

$$
\frac{\partial q}{\partial t}=\mu^{\prime}\left(\frac{\partial}{\partial x}\left(\alpha^{\prime} \frac{\partial F}{\partial x}\right)+\frac{\partial}{\partial y}\left(\alpha^{\prime} \frac{\partial F}{\partial y}\right)\right)-\gamma q
$$

where $F=q^{p+1}$. Note that Eqs. 9-10 are special cases of Eq. 8 . Thus, the numerical approach described here to solve Eq. 8 is also suitable to solve Eqs. 9-10. The Runge Kutta 4th order scheme for 1-D diffusion equation (2nd order central spacial difference) suffers CFL limit of 0.695 , whereas the trapezium formula is unconditionally stable. The above equation, discretized using trapezium formula (except for the source term) and coupled with central spacial difference, becomes:

$$
\begin{aligned}
q_{i, j}^{\mathrm{m}+1}-q_{\mathrm{i}, \mathrm{j}}^{\mathrm{m}}= & \frac{\Delta t}{2} \mu^{\prime}\left(\frac{\delta_{\mathrm{x}}^{-} \alpha^{\prime} \delta_{\mathrm{x}}^{+} F_{1, \mathrm{j}}^{\mathrm{m}+1}}{\Delta x^{2}}+\frac{\delta_{\mathrm{y}}^{-} \alpha^{\prime} \delta_{\mathrm{y}}^{+} F_{1, j}^{\mathrm{m}+1}}{\Delta y^{2}}\right. \\
& \left.+\frac{\delta_{\mathrm{x}}^{-} \alpha^{\prime} \delta_{\mathrm{x}}^{+} F_{1, \mathrm{j}}^{\mathrm{m}}}{\Delta x^{2}}+\frac{\delta_{\mathrm{y}}^{-} \alpha^{\prime} \delta_{\mathrm{y}}^{+} F_{1, \mathrm{j}}^{\mathrm{m}}}{\Delta y^{2}}\right)-\Delta t \gamma q_{\mathrm{i}, \mathrm{m}}^{\mathrm{m}}
\end{aligned}
$$

where $\mathrm{m}$ is the time step index, $\Delta x$ and $\Delta y$ are the grid spaces and $\delta_{x}^{+} F_{\mathrm{i}, \mathrm{j}}=F_{\mathrm{i}+1, \mathrm{j}}-F_{\mathrm{i}, \mathrm{j}}, \delta_{x}^{-} F_{\mathrm{i}, \mathrm{j}}=F_{\mathrm{i}, \mathrm{j}}-F_{\mathrm{i}-1, \mathrm{j}}$. Through linearlization and factorization, the equation can be written in $\Delta$-form:

$$
\begin{array}{r}
\left(1-\frac{\Delta t \mu^{\prime}}{2 \Delta x^{2}} \delta_{x}^{-} \alpha^{\prime} A_{i, j}^{\mathrm{m}} \delta_{x}^{+}\right)\left(1-\frac{\Delta t \mu^{\prime}}{2 \Delta y^{2}} \delta_{y}^{-} \alpha^{\prime} A_{1, j}^{\mathrm{m}} \delta_{y}^{+}\right) \Delta q \\
=\Delta t\left(\mu^{\prime}\left(\frac{\delta_{\mathrm{x}}^{-} \alpha^{\prime} \delta_{\mathrm{x}}^{+} F_{\mathrm{i}, \mathrm{j}}^{\mathrm{m}}}{\Delta x^{2}}+\frac{\delta_{\mathrm{y}}^{-} \alpha^{\prime} \delta_{\mathrm{y}}^{+} F_{1, \mathrm{j}}^{\mathrm{m}}}{\Delta y^{2}}\right)-\gamma_{q_{1, j}^{\mathrm{m}}}^{\mathrm{m}}\right),
\end{array}
$$

where $A=\mathrm{d} F / \mathrm{d} q=(\mathrm{n}+1) q^{\mathrm{n}}$ and $\Delta q=q^{\mathrm{m}+1)}-q^{\mathrm{m}}$. The codes have been written for generalized coordinates which allow nonuniform grid stretching and have been validated by solving model equations such as Poisson's equation, heat equation and $\partial^{2} u / \partial x^{2}=u$ The accuracy of the algorithm has been verified. For the paddle structure with holes, the pressure within holes is assumed ambient pressure. The simple one-zonal grid approach is used. In the hole regions, the right-hand side of Eq. 13 is masked to be zero; the corresponding diagonal term is set to be one, and off-diagonal terms are zeros. Once $q$ is obtained, the damping force $F_{\mathrm{d}}$ can be integrated to couple with the dynamic model.

The one-dimensional dynamic model for the electrostatically driven paddle structure above the substrate can be expressed as

$$
m \frac{\mathrm{d}^{2} h}{\mathrm{~d} t^{2}}+k\left(h-h_{\mathrm{i}}\right)=F_{\mathrm{e}}+F_{\mathrm{d}}
$$

where $m$ is mass, $k$ is spring constant, and $h_{\mathrm{i}}$ is the initial paddle-to-substrate gap with no spring force. The electrostatic force $F_{\mathrm{e}}=\varepsilon A \mathrm{~V}^{2} / 2 h^{2}$, where $\varepsilon$ is the permittivity and $\mathrm{V}$ is the applied voltage. The 4th order Runge-Kutta method is used in the time integration.

\section{RESULTS AND DISCUSSION}

In the present work, the initial displacement and velocity of the paddle are set to zero. The voltage is a step function applied at $t=0$. The permittivity $\varepsilon=8.9 \times 10^{-12} \mathrm{~F} / \mathrm{m}$; the area of the paddle, $A$, is approximated with $50 \times 100 \mu \mathrm{m}^{2}$ regardless of the hole area; $k$ is set to be $0.1 \mathrm{~N} / \mathrm{m}$; the mass of the paddle, $m$, is $1.15 \times 10^{-11} \mathrm{~kg}$; the gap between paddle and substrate is $5 \mu \mathrm{m}$. The grid point used here is $101 \times 51$ in the $x-y$ plane.

The effects of slip and no-slip boundary conditions are compared in Fig. 2. The diffusion reflection coefficient, $d$, used here is 0.9 and the mean free path, $\lambda$, of air is $0.0635 \mu \mathrm{m}$. Applying slip boundary condition effectively reduces the strength of the forcing term in the Poisson's equation, and consequently reduces the damping force in the dynamic system. When the applied voltage is $8.7 \mathrm{~V}$, two stable steady-state solutions exist. These two stable solutions are qualitatively illustrated in Fig. 3(a), labeled " $A$ " and "B", with the critically stable solutions illustrated in Fig. 3(b). In the stable solution " $A$ ", as one reduces the gap between the paddle and the substrate, i.e., moving towards the right on the $x$ axis in Fig. 3(a) away from point " $\mathrm{A}$ ", the spring back force becomes greater than the electrostatic force $F_{\mathrm{e}}$ for a fixed applied voltage, and thus pulling back the paddle structure to point " $A$ ", making point " $A$ " a stable solution. With the same analogy, it is obvious that point " $C$ " is an unstable solution. As one further increases the applied voltage, the electrostatic force curve in Fig. 3 shifts upward, forcing point " $A$ " and " $C$ " move closer. When reaching a critical voltage, point " $A$ " merges with point " $C$ ", and makes that a critically stable solution as illustrated in Fig. 3(b). After passing this critical voltage, the cantilevered paddle structure will converge to the only remaining stable steady-state solution labeled " $B$ " in Fig. 3. This jump from the critically stable solution $(A / C)$ to the stable solution " $B$ " is commonly observed as the "snapping" motion in the parallel plate system, and occurs at one-third the distance of the total, unforced separation gap. 


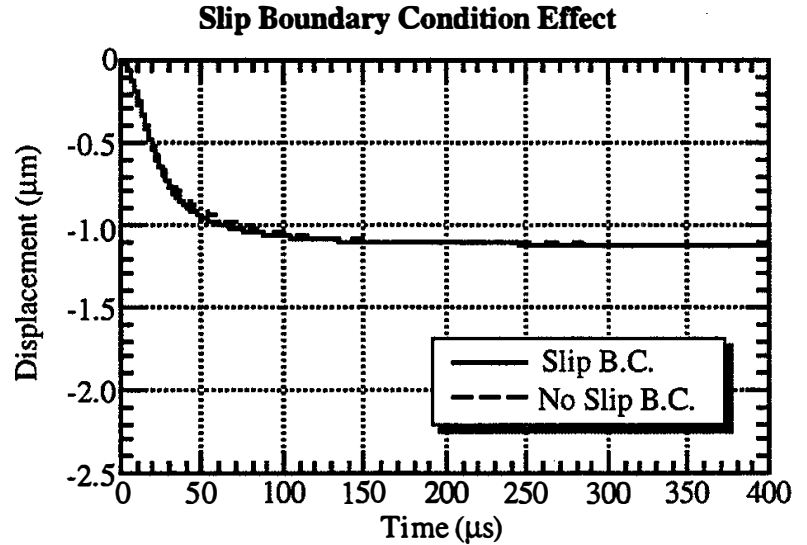

(a)

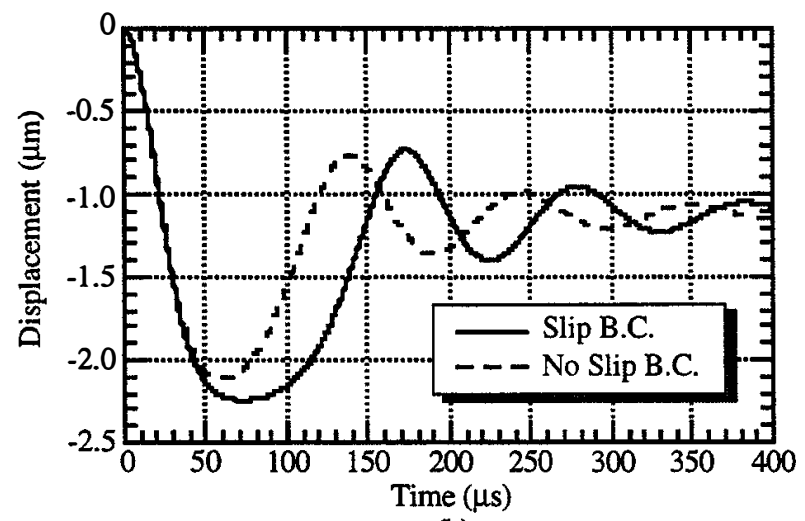

(b)

Figure 2. Simulation results showing the effect of slip boundary condition for the over-damped (no-hole paddle) case in $(a)$ and for the under-damped (8-hole paddle) case in (b).

Using current initial condition, the results in Fig. 2 show that the paddle converges to the solution with smaller displacement (Point " $A$ " in Fig. 3). By further reducing the diffuse reflection coefficient, e.g., from 0.9 to 0.8 , or increasing $\lambda$, the damping force is reduced, and the paddle motion in response to the step input has been observed to converge to the other branch solution " $B$ " and hit the substrate.

When the same voltage of $8.7 \mathrm{~V}$ is applied to various structural designs (no-hole, two-hole and eight-hole), the paddle can move with no-oscillation, one-oscillation, and underdamping oscillation as shown in Fig. 4. The corresponding contours of the pressure load when the transient damping force is at its maximum are shown in Fig. 5. The lighter contour color denotes lower pressure. The maximum pressure always occurs remote to the hole and to the paddle fringe edges. The maximum pressure is $1.000186 p_{\mathrm{a}}$ for the no-hole paddle, $1.000168 p_{\mathrm{a}}$ for the 2 -hole paddle, and $1.00008 p_{\mathrm{a}}$ for the 8 -hole paddle. The results indicate that the holes effectively relieve the pressure buildup in the gap, and thus reduce the damping force.

For the example system described in this paper, the critical voltage occurs at $9.123 \mathrm{~V}$, at which one of the two stable steady-state solutions (point " $A$ ") merges with the unstable solution (point "C"), and only one stable steady solution (point " $B$ ") remains. Therefore, the paddle structure moves across the separating gap and comes in contact with the substrate. The transient behaviors of the no-hole design and of the 8-hole design are compared in Fig. 6 with an applied voltage of $9.124 \mathrm{~V}$. The slow contact of the no-hole paddle is dramatically improved with the 8-hole design. The contact time is reduced from $8.9 \mathrm{~ms}$ to $67 \mu \mathrm{s}$, a more than 100 times reduction. The experimental data has qualitatively verified these results [6], and Fig. 7 shows two SEM images of a test structure.
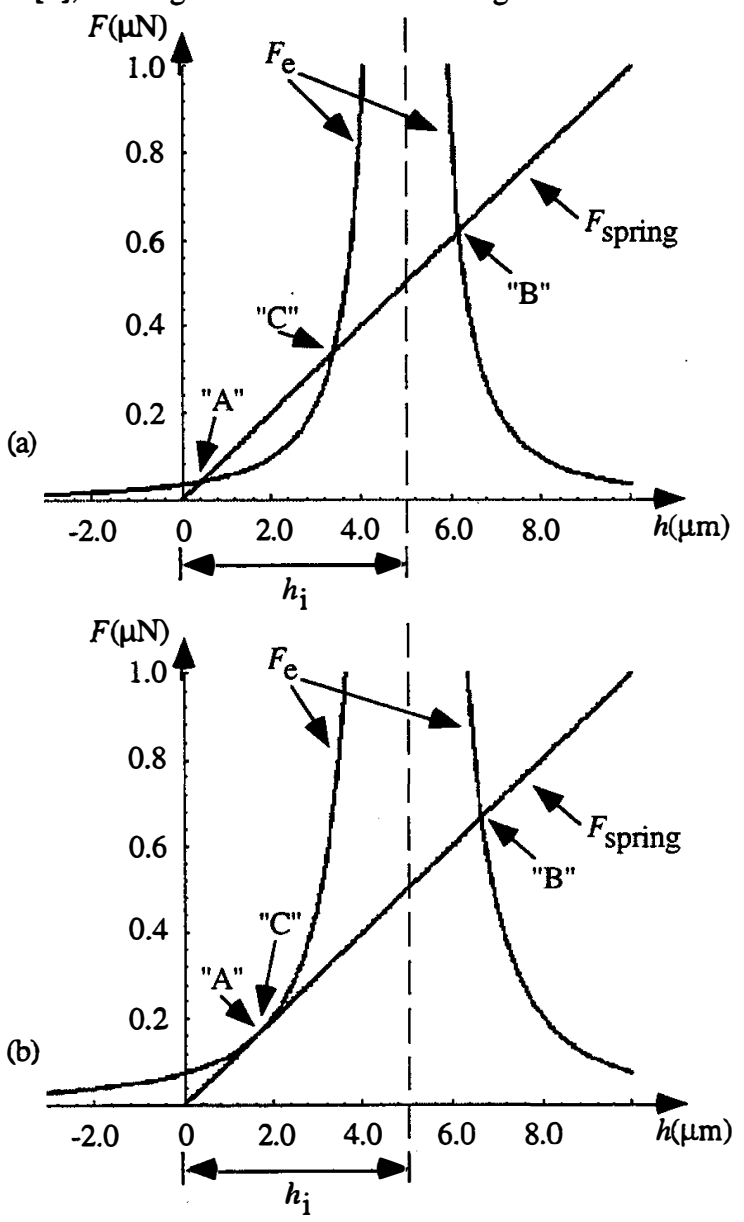

Figure 3. Plots of the electrostatic force and the spring-back force as a function of the separating gap between two parallel electrodes showing the two stable solutions " $A$ " and " $B$ ", and one unstable solution " $C$ ". As the points " $A$ " and " $C$ " merge, shown in (b), the solution becomes critically stable.

Dynamic Response to a Step Input

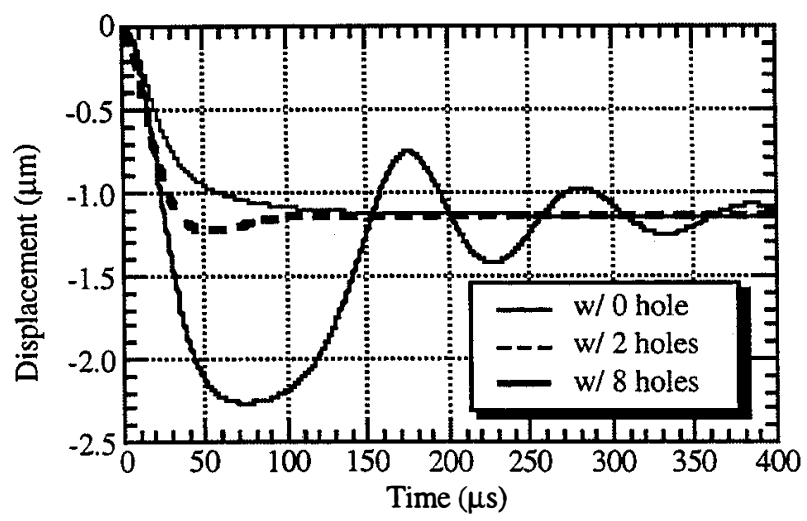

Figure 4. Effect of structural design in terms of the number of holes used in the cantilevered paddle structure on the paddle's dynamic response to a step voltage input. 
(a)

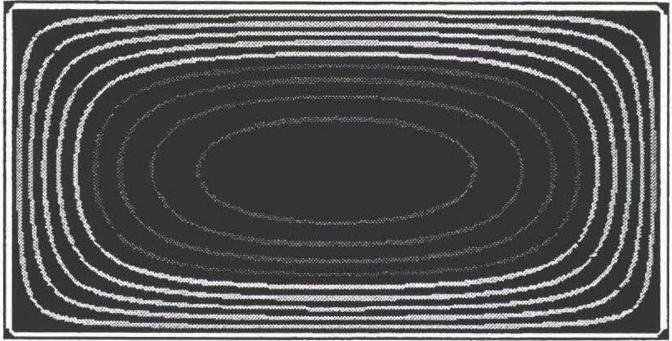

(b)

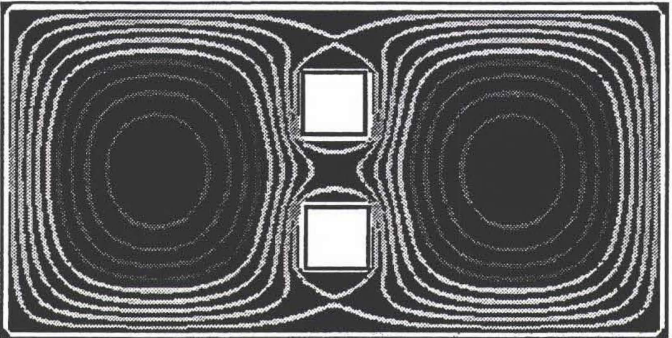

(c)

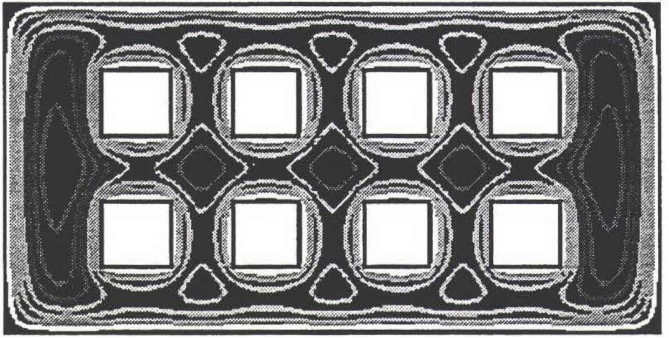

Figure 5. Contours of pressure loads at maximum transient damping force for designs with (a) no-hole, (b) 2-hole, and (c) 8-hole. The dynamic responses of these designs are in Fig. 4.

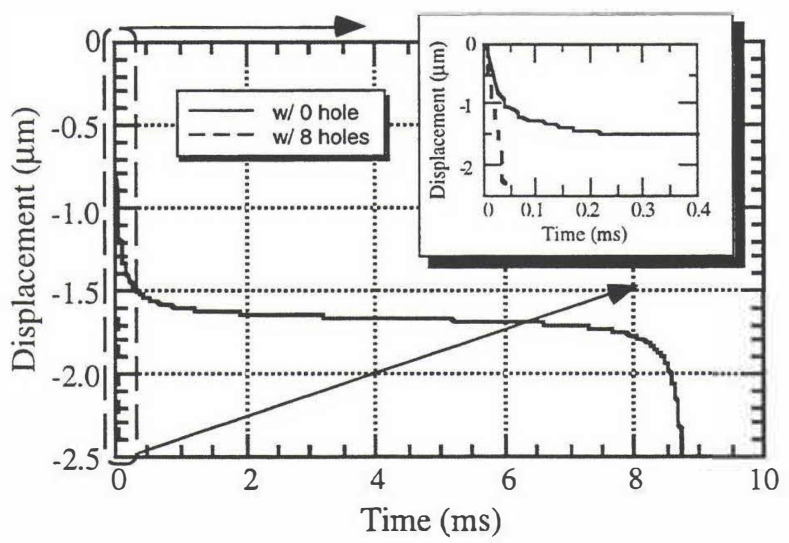

Figure 6. Simulation results showing the transient responses of the no-hole design and the 8-hole design with a step function of $9.124 \mathrm{~V}$ applied. The contact time has been reduced from $8.9 \mathrm{~ms}$ (in the no-hole design) to $67 \mu \mathrm{s}$ (in the 8-hole design), a more than 100 times reduction.

\section{CONCLUSION}

Software for simulating the dynamics and the damping effects of MEMS squeeze type devices has been developed. The micro-fluidynamic damping effect is modeled to cover the slip flow by solving the Reynolds equation coupled with slip boundary condition at the structure surfaces. The analysis and the numerical procedure are presented. The software is very well-suited for modeling squeeze type devices with various holes, and it can be very effective for designing new MEMS squeeze type devices with desired damping characteristics.
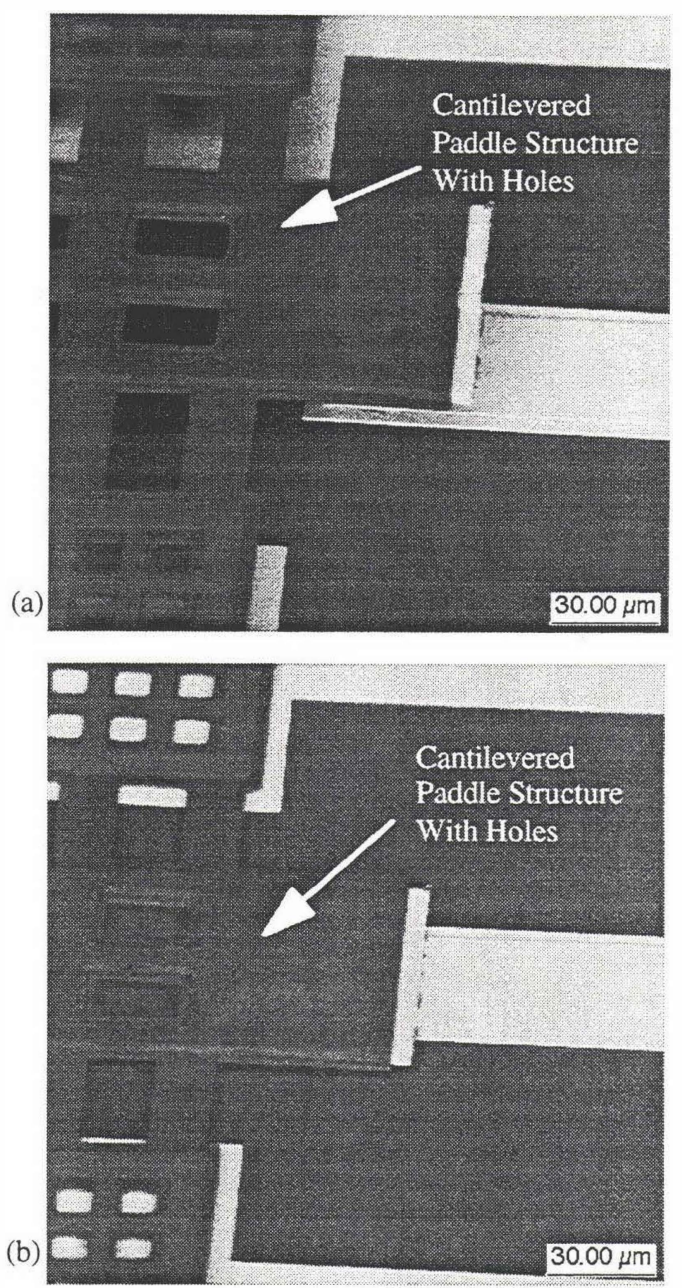

Figure 7. SEM back scattering images showing a close-up, tilted view of a test paddle structure with holes in (a) Up and (b) Down positions relative to the substrate.

\section{REFERENCES}

1. A. Beskok and G. E. Karniadakis, "Simulation of SlipFlows in Complex Micro-Geometries," ASME, DSC-Vol. 40 (1992), pp. 355-370.

2. E. S. Piekos and K. S. Breuer, "DSMC Modeling of Micromechanical Devices," AIAA-95-2089 (1995).

3. W. S. Griffin, H. H. Richardson, and S. Yamanami, “A study of Fluid Squeeze-Film Damping," Journal of Basic Engineering (1966), pp. 451-456.

4. L. Zhang, D. Cho, H. Shiraishi, and W. Trimmer, "Squeeze Film Damping in Microelectromechanical Systems," ASME, DSC- Vol. 40 (1992), pp. 149-160.

5. S. A. Schaaf and P. L. Chambre, "Flow of Rarefied Gases," Section H in Fundamentals of Gas Dynamics, H. W. Emmons, ed., Princeton University Press (1958), p. 718.

6. J. J. Yao and M. F. Chang, "A Surface Micromachined Miniature Switch for Telecommunications Applications With Signal Frequencies From DC Up To $4 \mathrm{GHz}$," in Tech. Digest, The 8th International Conference on Solid-State Sensors and Actuators, Transducers-95, June 25-29, 1995, Stockholm, Sweden, pp. 384-387. 